biological agent, dose and adherence were examined. To calculate the adherence we used a record of prescriptions dispensed over a period of six months. We used the formula: \% adherence $=$ no. of units dispensed/no. of units theoretically needed $\times 100$.

Results The sample included 62 patients, 45 males and 17 females with mean age of 50 years (range 12-81). 53.2\% were using etanercept, $43.6 \%$ adalimumab and $3.2 \%$ infliximab. The adherence was high in the infliximab group (94\%) and very similar in the other groups (etanercept $83.7 \%$, adalimumab $87.4 \%$ ). In the adalimumab cohort $11 \%$ had a reduced dose, in the etanercept group 9\% had a reduced and $30 \%$ an increased dose. In all these groups the calculated adherence was quite similar.

Conclusions As described in the literature, adherence to biologicals was significantly higher compared with the adherence observed with other treatments for psoriasis. Infliximab had the highest rate, maybe because it is administered in hospital. There was no difference between adalimumab and etanercept. It is known that there is progressive loss of patient adherence to treatment, for this reason is important to focus the attention on this concept.

No conflict of interest.

\section{OHP-035 EVALUATING SINGLE-INCISION SLINGS IN FEMALE STRESS URINARY INCONTINENCE: THE USEFULNESS OF THE CONSORT STATEMENT CRITERIA}

doi:10.1136/ejhpharm-2013-000276.409

$\underline{{ }^{1} \mathrm{~S} \text { Diallo, }}{ }^{1} \mathrm{~A}$ Josephson, ${ }^{2} \mathrm{~F}$ Cour, ${ }^{2} \mathrm{~A}$ Vidart, ${ }^{2 \mathrm{H}}$ Botto, ${ }^{2 \mathrm{~T}}$ Lebret, ${ }^{1 B}$ Bonan. ${ }^{1} \mathrm{Hôpital}$ Foch, Pharmacy, Suresnes, France; ${ }^{2}$ Hôpital Foch, Urology, Suresnes, France

Background Unlike drugs, medical devices (MDs) are not submitted for health authority marketing authorization based on in-depth clinical evaluation: critical review on an evidence-based medicine approach is essential for practitioners. The Consolidated Standards of Reporting Trials (CONSORT) statement is an international consensus expert guideline aimed at improving the reporting quality of clinical trials reports.

Purpose To evaluate the usefulness and applicability of the CONSORT for journal articles reporting randomised controlled trials (RCTs) evaluating an implantable $\mathrm{MD}$.

Materials and Methods Original articles published before 2012 reporting RCTs assessing single-incision slings (SISs) in the treatment of female stress urinary incontinence were searched for in PubMed and Embase databases. Reporting quality was assessed by two hospital pharmacists and two urological surgeons according to three CONSORT checklists: abstract (17 items), standard (37 items) and extension for non-pharmacological trials (20 items); the results were discussed to reach a consensus.

Results Among 135 articles retrieved, eight articles met the inclusion criteria and were assessed. Abstract scores ranged from 4.7 to 14.1 out of 20 . Standard scores were greater than 10.0 out of 20 for most articles; the extension scores did not exceed 5.0 out of 10 . Half the reported trials were not identified as randomised in the title. Three articles did not mention any confidence interval or standard deviation for outcomes. The interventions were incompletely described; only four articles reported the configuration of the devices. Four articles reported whether blinding was achieved but lack of blinding was never discussed as a potential source of bias. Few articles reported the operators and centres' characteristics and their impact on statistical analysis.

Conclusions The reporting quality of SISs RCTs should be improved because readers require complete, clear and transparent information to assess the relevance and applicability of results. Our study supports further use of the CONSORT criteria to enhance and assess the reporting quality of surgical trials.

No conflict of interest.

\section{OHP-036 EVALUATION OF ANTIBIOTIC APPRIOPRIATENESS AND USE IN IMOLA HOSPITAL}

doi:10.1136/ejhpharm-2013-000276.410

MC Silvani, L Paternoster, S Calabria, I Martens, S Gambetti. AUSL Imola, Assistenza Farmaceutica, Imola (Bologna), Italy

Background Misuse of antibiotics in hospitals may cause bacterial resistance as well as increased costs and unnecessary exposure of patients to drugs.

Purpose To evaluate antimicrobial consumption and appropriateness through a new antibacterial stewardship policy.

Materials and Methods The study was carried out in Imola Hospital (Bologna) and 2009-2011 drug consumption data were obtained from the pharmacy service. Data were analysed by clinical area and single wards and were expressed by ATC classification and defined daily doses per 100 bed-days (DDD). A form for personalised antibacterial treatment (ATf), including diagnosis and documented reasons for the choice of antibiotic, was introduced for levofloxacin, teicoplanin, meropenem, linezolid, tigecycline and daptomycin.

Results In 2011, overall antibacterial consumption was 78 DDD (+4\% vs. 2010); the major increase was observed in medical units (MED: $+9 \%$ ) and paediatric/gynaecological units $(+6 \%)$. Intensive care units/emergency department (ICUs/EDs) and surgical units (SUR) exhibited a decrease in consumption $(-13 \%,-7 \%$, respectively). The use of critical antimicrobial agents decreased: fluoroquinolones (19 DDD, -15\%), carbapenems (3.5 DDD, -18\%) and glycopeptides (3.1 DDD, $-17 \%$ ). The introduction of ATfs (May 2011) contributed to a decrease in the consumption of antibiotics (e.g. MED: 75 DDD semester I vs. 71 DDD semester II 2011; overall 2011: 73 DDD). The analysis of ATfs shows that critical antibacterial agents were mainly prescribed to treat respiratory tract infections (MED: 58\%, ICU/ED: 44\%, SUR 30\%), urinary tract (MED e ICU/ ED: 20\%), skin and soft tissues (SUR: 35\%, ICU/ED: 16\%, MED: 6\%) and intra-abdominal infections (SUR: $9 \%$ ). Levofloxacin (55\%) and meropenem $(11 \%)$ were the most prescribed for respiratory tract infections, teicoplanin (6\%) for skin and soft tissue infections.

Conclusions Our stewardship policy led to a reduction in the use of wide-spectrum antibiotics, so ATf may represent a valid method of rationalising the choice of antimicrobial treatment.

Acknowledgements Agenzia Italiano Del Farmaco, Emilia Romagna Region.

No conflict of interest.

\section{OHP-037 EVALUATION OF CHANGE OF ETANERCEPT SUBCUTANEOUS ADMINISTRATION DEVICE}

doi:10.1136/ejhpharm-2013-000276.411

${ }^{1} \mathrm{MJ}$ Gayan Lera, ${ }^{1} \mathrm{M}$ Ercilla Liceaga, ${ }^{1} \mathrm{C}$ Ripa Ciaurriz, ${ }^{2} \mathrm{C}$ Sarasqueta Eizaguirre, ${ }^{1} \mathrm{I}$ Barral Juez, 'MD Mauleon Echeverria, "I Aguirre Zubia, 'P Pascual Gonzalez, 'P Carmona Oyaga, 'M Umerez Igartua. 'Donostia University Hospital, Pharmacy, San Sebastián, Spain; ${ }^{2 B i o d o n o s t i a ~}$

Background Etanercept is a soluble tumour necrosis factor receptor fusion protein used in a variety of arthropathies. A new administration device (pen) has recently been marketed.

Purpose To evaluate pain differences and preference between the etanercept syringe and pen as well as the relation between pain and demographic and anthropometric factors.

Materials and Methods All patients with the etanercept pen from 1 January 2012 to 31 March 2012 who had previously used the syringe were chosen. Gender, age, Body Mass Index (BMI), diagnosis, self-administration, pain perception $(0=$ no pain; $10=$ maximum pain) and device preference were recorded. Statistical analysis: Student's t-test and variance analysis were used for comparisons of means, chi-square and Fisher's test for proportions, and nonparametric tests for pain. 
Results 109 patients (43\% men; 57\% women) met inclusion criteria. Mean age was $54 \pm 13.5$ years and mean BMI $26.5 \pm 4.8 \mathrm{~kg} / \mathrm{m}^{2}$. $58.7 \%$ had Rheumatoid Arthritis, 19.3\% Ankylosing Spondylitis, 1.8\% Juvenile Idiopathic Arthritis, 16.5\% Psoriatic Arthritis and $3.7 \%$ Psoriasis. $82 \%$ self-administrated the pen, and $71 \%$ the syringe. The median pain with the syringe was 3 [interquartile range (IOR): $2-6]$ and with the pen was 4 [IOR: $2-5$ ] $(\mathrm{P}=0.008)$. 65\% reported the same pain with both devices. $35 \%$ reported differences in pain and most of them $(71 \%)$ had much pain $(>5)$ with the pen and little pain $(<5)$ with the syringe.

There was a statistically significant association of pain with gender: women had more pain with the pen $(P=0.03)$, but less with the syringe $(p>0.05)$. There was no association with BMI, age or diagnosis. $59 \%$ preferred the pen, $25 \%$ the syringe, and $16 \%$ did not mind.

Conclusions An association of pain with pen device and female gender was found. However there was no association with BMI, age or diagnosis. Acceptance of the pen and self-administration were higher even though pain was greater, so it is necessary to maintain both devices to assure adherence.

No conflict of interest.

\section{OHP-038 EVALUATION OF QUALITY OF LIFE IN PATIENTS WITH MULTIPLE SCLEROSIS}

doi:10.1136/ejhpharm-2013-000276.412

'MC Izquierdo Navarro, 'C Matallana Martin, 'V Martínez Santana, ${ }^{2 M A}$ Calleja Hernandez, 'AM López González, 'MT Sánchez Sánchez. 'Hospital Clínico Universitario, Pharmacy Department, Valladolid, Spain; ${ }^{2}$ Hospital Vírgen de la Nieves, Pharmacy Department, Granada, Spain

Background Several studies have evaluated quality of life (QoL) by filling in the EuroQoL-5D..In most of them, it is found that the two dimensions of EuroQoL-5D most associated with a poor QoL are pain/discomfort and anxiety/depression.

Purpose To find the dimensions of EuroQoL-5D that are more frequently associated with $\mathrm{QoL}$ in patients diagnosed with RelapsingRemitting Multiple Sclerosis (RRMS).

Materials and Methods Observational, four-month, cross-sectional study (January-April 2012) to assess QoL in patients diagnosed with RRMS.

Sex, age and Expanded Disability Status Scale (EDSS) were gathered from Pacientes Externos (Farmatools programme 2.4 version).

Patients who filled in the EuroQoL-5D returned it to the pharmacy service.

Results 84 patients were included; 62 completed the questionnaire.

Mean age was $36.94 \pm 8.67 .65 .47 \%$ of patients were women, $34.52 \%$ were men. The mean EDSS was $2.03 \pm 1.50$.

The survey results of the questionnaire broken down by items were:

\section{Abstract OHP-038 Table 1}

\begin{tabular}{llll}
\hline & & \multicolumn{2}{c}{ Number } \\
\hline Mobility & I have no problems walking & 42 & 67.7 \\
& I have some problems & 20 & 32.3 \\
Personal Care & I am confined to bed & 0 & 0 \\
& I have no problems with self-care & 56 & 90.3 \\
& I have some problems & 6 & 9.7 \\
Usual activities & I am unable to wash or dress myself & 0 & 0 \\
& I have no problems with performing my usual activities & 42 & 67.7 \\
Pain/discomfort (P/D) & I have some problems & 20 & 32.3 \\
& I am unable to perform my usual activities & 0 & 0 \\
Anxiety/Depression & I have no P/D & 28 & 45.2 \\
& I have moderate P/D & 33 & 53.2 \\
& I have extreme P/D & 1 & 1.6 \\
& I am mot anxious or depressed (A/De) & 28 & 45.2 \\
& I am extremely A/De & 28 & 45.2 \\
\hline
\end{tabular}

The mean value obtained in the questionnaire was $0.71 \pm 0.19$. Conclusions As has been shown in previous studies, the two dimensions of EuroQoL-5D that most affected the QoL were pain/ discomfort and anxiety/depression.

No conflict of interest.

\section{OHP-039 EXPANDING THE INVOLVEMENT OF PHARMACY SERVICES VIA COMPUTERISED MEDICAL FILES}

doi:10.1136/ejhpharm-2013-000276.413

${ }^{1} \mathrm{~N}$ Mansur, ${ }^{1} \mathrm{~T}$ Gruenewald, ${ }^{2} \mathrm{E}$ Sporta, ${ }^{3} \mathrm{M}$ Shindler, ${ }^{3} \mathrm{D}$ Lavi. ${ }^{1}$ Beilinson Hospital, Pharmacy services, Tel Aviv, Israel; 'Beilinson Hospital, Computer Services/Elad Software Systems, Tel Aviv, Israel; ${ }^{3 B}$ Beilinson Hospital, Computer Services, Tel Aviv, Israel

Background Pharmacists are essential for the safe use of medicines, and have a very important role in providing comprehensive drug management. Their crucial responsibilities in medicines management and promoting quality control necessitate developing a computerised tool to improve their communication with other medical team members.

Purpose To develop a pharmacist interface, as a part of the computerised medical file 'Chameleon', to display all the information required by pharmacists for preparing and documenting their intervention.

\section{Materials and Methods}

Step 1: mapping the processes required for implementation of the system

Step 2: preparing a dedicated tool with two components:

1. A pharmacist interface: a screen designed to show all related data required for a clinical pharmacist to form his opinion regarding the medicinal treatment. The pharmacist intervention is documented in an assigned field 'pharmacist follow up', which is also displayed beside the 'physician follow up' field in the physician interface to save switching screens.

2. The pharmacy services as an advisory ward: the pharmacists' team is defined as an advisory ward that can be invited by the physicians. Requests for advice are displayed in a pharmacist work list.

Results The pharmacist interface was integrated into the "Chameleon' and is used regularly. It is a convenient tool that displays all the information required for a professional pharmacist's opinion, and improves medical team communication by allowing this opinion to be viewed by other staff members. There is an ongoing process of assimilation and dissemination of the computerised availability of pharmacy advisory services. There are two topics in development: (a) physician feedback and reference regarding the pharmacist advice, and (b) the ability to monitor all revised cases.

Conclusions The computerised tool satisfies the pharmacist work process and improves communication with the medical staff. The final tool will generate statistics about its contribution to medical personnel and improve the quality of pharmacy services in this medical care hospital.

No conflict of interest.

\section{OHP-040 FINANCIAL ASSESSMENT OF INTRAVENOUS MIXTURE PREPARATION}

doi:10.1136/ejhpharm-2013-000276.414

M Uriarte, MJ Agustín, V Gimeno, I Larrodé, 0 Pascual, P Palomo, MR Abad. Miguel Servet Hospital, Pharmacy Service, Zaragoza, Spain

Background Intravenous treatment is expensive so we studied two different working options.

Purpose To evaluate the savings that preparing intravenous mixtures centrally in the pharmacy service hypothetically made in 2011 in our hospital. 